\title{
Region-based Active Contour Segmentation Model with Local Discriminant Criterion
}

\author{
F.Z. Zhao ${ }^{* 1}$, H.Y. Liang ${ }^{2}$, X.L.Wu ${ }^{3}$ and D.H. Ding ${ }^{4}$ \\ ${ }^{1,2,3}$ School of Mathematics \& Computer Science, Hezhou University, China, \\ Guangxi \\ ${ }^{4}$ School of Computer Science \& Information Engineering, Hunan University of \\ Arts and Science, China, Hunan \\ ${ }^{1}$ :gxgzfz@163.com, 2haiyingliang@126.com, ${ }^{3}$ wuxianglin2015@163.com, \\ ${ }^{4}$ dingdehong@qq.com
}

\begin{abstract}
This paper presents a novel region-based active contour model for image segmentation in a variational level set formulation. We define a local discriminant criterion on the basis of the global and local region-based active contour model. The objective function in this model is thereafter minimized via level set method. By introducing the local discriminant criterion to separate background and foreground in local regions, our model not only achieves accurate segmentation results, but also is robust to initialization. Extensive experiments are reported to demonstrate that our method holds higher segmentation accuracy and more initialization robustness, compared with the global region-based and local region-based methods. Experimental results for synthetic images and real medical images show desirable performances of our method.
\end{abstract}

Keywords: active contour model; image segmentation; local discrimination criterion

\section{Introduction}

Image segmentation has been widely applied in computer vision, object tracking, medical imaging, and geographic imaging $[1,2]$. Typically, it is a process in which the image is divided into a series of non-intersected sub-regions with approximately similar properties. Many techniques on this problem have been proposed in the literature, such as region-growing, clustering, active contour model, and so forth. However, the segmentation problem is still an important and challenging research topic due to the complexity of images.

Among those segmentation methods, active contour model (ACM) gets more attention in image segmentation field. The existing active contour model can be divided into two categories: parameter active contour models [3,4] and geometric active contour models[5,6]. Parametric active contour models employ an energy functional minimization formulation to search for the correct location for a parameterized contour. Hence, they are more suitable for segmentation of a single structure in an image. Geometric active contour models use level set method to find the locations of final contours. They are more suitable for segmentation of multiple structures in an image due to its robustness in merging and splitting the contours during evolution. The geometric active contour models can be roughly categorized into two classes: edge-based models [7] and region-based models $[8,9]$.

In general, edge-based models typically use image gradient information as an imagebased force to attract the contour toward object boundaries. These models have been successfully used for general images with strong object boundaries, but they may suffer 
from boundary leakage problem for the medical images, which typically contain weak boundaries due to low contrast and partial volume effect. Region-based models have better performance than edge-based models in the presence of weak boundaries. However, region-based models tend to rely on intensity homogeneity. For example, the well-known Chan-Vese(CV) model [10] is based on the assumption that image intensities are statistically homogeneous in each region, and therefore it fails to segment the images with intensity inhomogeneity.

Recently, Li et al. proposed a local binary fitting (LBF) model [11] to overcome the difficulty in segmentation caused by intensity inhomogeneity. The LBF model draws upon spatially varying local region information and thus is able to deal with intensity inhomogeneity. Some related methods were recently proposed in [12-15], which had similar capability of handling intensity inhomogeneity as the LBF model. These methods are, however, to some extent sensitive to initialization, which limit their practical applications. Besides, in such models, the energy functions are classically transformed into a level set formulation and then minimized by solving the corresponding EulerLagrange equations, which are slow to converge.

To improve the robustness to initialization, some hybrid models combining the local and global intensity fitting energies were proposed [16,17]. In [16], the authors defined an energy function (Local and Global Intensity Fitting, LGIF) with a local intensity fitting term used in LBF model and an auxiliary global intensity fitting term used in C-V model. However, the LGIF model also has several deficiencies as follows. Firstly, the nonconvexity of the LGIF energy functional may cause local minima. Secondly, for different images, it is necessary to choose appropriate weight values to control the local and global intensity fitting force. Thirdly, the segmentation result is still slightly dependent on the location of the initial contour, because the weight coefficient between the local term and the global term is a fixed positive constant.

In this paper, motivated by the CV, the LBF and the LGIF model, we propose the ILGIF (Improved Local and Global Intensity Fitting) model which overcomes the above mentioned deficiencies of the LGIF model. The fitting energy function of the ILGIF model is established by introducing the local criterion information on the basis of the LGIF model. Our new model has the following advantages over the LGIF model. First of all, by applying the criterion information, we define a new energy functional to guarantee that the new algorithm has the tendency to compute a global minima. Secondly, our new model can control the influence of the local and global region information and criterion information by itself. Finally, our new model is not sensitive to initial position. We apply this method to synthetic images and real-world images, and the experimental results verify the validity of the method in this paper.

The reminder of this paper is organized as follows. In Section 2, we review some wellknown region-based models and their limitations. The proposed method is introduced in Section 3. The implementation and results are given in Section 4. Some conclusion is given in Section 5.

\section{Background}

\subsection{The CV Model}

Chan and Vese proposed CV model, which assumed that the original image was a piecewise constant function. For an image $I$ on the image domain $\Omega$, they proposed to minimize the following energy: 


$$
\begin{gathered}
E^{C V}\left(c_{1}, c_{2}, C\right)=\lambda_{1} \int_{\text {inside }(C)}\left|I(x)-c_{1}\right|^{2} d x \\
\quad+\lambda_{2} \int_{\text {outside }(C)}\left|I(x)-c_{2}\right|^{2} d x+v|C|
\end{gathered}
$$

(1)

Where inside $(C)$ and outside $(C)$ represent the region inside and outside of the contour $C$, respectively, and $c_{1}$ and $c_{2}$ are two constants that approximate the image in inside $(C)$ and outside $(C)$, respectively. $\lambda_{1}, \lambda_{2}$ and $v$ are nonnegative constant.

The CV model has good performance in image segmentation due to its ability of obtaining a larger convergence range and being less sensitive to the initialization. However, if the intensities with inside $C$ or outside $C$ are not homogeneous, the constants will not be accurate. As a consequence, the $\mathrm{CV}$ model generally fails to segment images with intensity inhomogeneity. Similarly, more general piecewise constant models in a multiphase level set framework are not well at such images either.

\subsection{The LBF Model}

To overcome the difficulty caused by intensity inhomogeneity, Li et al. proposed the LBF model, which could segment images with intensity inhomogeneity, using the local intensity information efficiently, and had achieved promising results. The data fitting term is defined in the form of kernel function. They proposed to minimize the local binary fitting energy

$$
\begin{aligned}
& E^{L B F}\left(f_{1}(x), f_{2}(x), \phi(x)\right)=\lambda_{1} \int\left[\int K_{\sigma}(x-y)\left|I(y)-f_{1}(x)\right|^{2} d y\right] d x \\
& +\lambda_{2} \int\left[\int K_{\sigma}(x-y)\left|I(y)-f_{1}(x)\right|^{2} d y\right] d x+v L(\phi(x))+\mu P(\phi(x))
\end{aligned}
$$

Where $\lambda_{1}, \lambda_{2}, v$ and $\mu$ are weighting positive constants. $K_{\sigma}$ is the Gaussian kernel function, and $\sigma$ is a constant to control the local region size. The first and second term are data fitting energy which drives evolution of the level set function. The third term is the length term to smooth the contour and the forth term is the level set regularization term to penalize the deviation of the level set function $\phi$ from a signed distance function.

Because of using local region information, specifically local intensity mean, the LBF model is able to provide desirable segmentation results even in the presence of intensity inhomogeneity. However, the LBF model is sensitive to initialization to some extent and is easy to fall into local minimum, which limits its practical applications.

\subsection{The LGIF Model}

Wang et al., proposed a LGIF model by combining the CV model and LBF model. The energy function of the LGIF model was defined as follows:

$$
\begin{array}{r}
E^{L G I F}\left(\phi, f_{1}, f_{2}, c_{1}, c_{2}\right)=(1-\omega) \cdot E^{C V}\left(\phi, c_{1}, c_{2}\right) \\
+\omega \cdot E^{L B F}\left(\phi, f_{1}, f_{2}\right)+v \cdot L(\phi)+\mu \cdot P(\phi)
\end{array}
$$

Where $\omega$ is a constant $(0 \leq \omega \leq 1) . v$ and $\mu$ are weighting positive constants. $E^{C V}\left(\phi, c_{1}, c_{2}\right)$ is the energy function of $\mathrm{CV}$ model, and $E^{L B F}\left(\phi, f_{1}, f_{2}\right)$ is the energy function of LBF model. $L(\phi)$ is length term and $P(\phi)$ is regularization term.

The technique of using global image information can improve the robustness to initialization of contours. However, when the evolving curve is close to object boundaries, 
the interference from the global intensity force will result to the deviation of contour from the real object boundary. At the same time, the double contours will appear and the edge position becomes blurred and inaccurate in the LGIF model. Furthermore, the LGIF model can be taken as the CV model acting on the image which is a combination of the original image and its transformed. To some extent, the LGIF model is also a CV model. So, it is difficult for the LGIF model to satisfactorily segment the image with intensity inhomogeneity.

\section{The Proposed Model}

In this section, we first introduce the local discriminant criterion. Then, the local discriminant criterion is integrated into the level set framework. Finally, we present the implementation details of our model. Besides, we will detail region-based active contour segmentation model with local discriminant criterion (ILGIF) for image segmentation.

\subsection{The Definition of Criterion}

As for it, we introduced discriminant criterion which is similar to Fisher criterion in expression form. The classify principle can be described as improving collection degree of within classification and separation degree of between classification. In theory, the Fisher criterion can obtain the best classification model, and it is suitable for the problem of classification. Therefore, we introduce a local criterion to our model.

The local criterion of this paper is defined as follows:

$J=\frac{\frac{1}{n_{1}} \int\left(I(x)-f_{1}\right)^{2} d x+\frac{1}{n_{2}} \int\left(I(x)-f_{2}\right)^{2} d x}{\left(f_{1}-f_{2}\right)^{2}}$

(4)

$n_{1}, n_{2}, f_{1}, f_{2}$ are updated after the iteration of level set function according to:

$\left\{\begin{array}{l}n_{1}=\frac{1}{\int_{\Omega} K_{\sigma} * H(\phi) d x d y} \\ n_{2}=\frac{1}{\int_{\Omega} K_{\sigma} *[1-H(\phi)] d x d y}\end{array}\right.$

(5)

$\left\{\begin{array}{c}f_{1}=\frac{\int_{\Omega} K_{\sigma} *[I(x) \cdot H(\phi)] d x d y}{\int_{\Omega} K_{\sigma} * H(\phi) d x d y} \\ f_{2}=\frac{\int_{\Omega} K_{\sigma} *[I(x) \cdot(1-H(\phi))] d x d y}{\int_{\Omega} K_{\sigma} *[1-H(\phi)] d x d y}\end{array}\right.$

(6)

Where $K_{\sigma}$ is the Gaussian kernel function, and $H(\phi)$ is the Heaviside function.

\subsection{The Definition of Energy Function}

On the basis of the LGIF model, we introduce the local discriminant criterion to the LGIF model and get the improved model of this paper, namely ILGIF model. The energy function is defined as follows: 


$$
\begin{gathered}
E\left(\phi, c_{1}, c_{2}, f_{1}, f_{2}\right)=\left[(1-\omega) E_{G}+\omega E_{L}\right]+ \\
\kappa J\left(\phi, f_{1}, f_{2}\right)+\nu \cdot L(\phi)+\mu \cdot P(\phi)
\end{gathered}
$$

(7)

Where $\phi$ is the level set function. $\omega$ is a constant $(0 \leq \omega \leq 1)$ and $\kappa$ is a constant $(0 \leq \kappa \leq 1) . v$ and $\mu$ are nonnegative constant. The first term is the data fitting term which drives the level set function to evolve. The second term is the criterion term. The third term is the length term. The fourth term is the regularization term.

According to different characteristics of segmentation images, the choice of the weighting coefficient of each item is different, which can be summarized as: if the initialized contour is away from the target boundary or image intensity is homogeneous, the CV model fitting term will be regarded as main power, and the parameter value $\omega$ should be chosen small enough; if the images are corrupted by intensity inhomogeneity, the LBF data fitting term will be regarded as main power, and the parameter value $\omega$ should be chosen large enough.

The length term and the regularization term are defined respectively as follows:

$$
\begin{aligned}
& L(\phi)=\int_{\Omega}|\nabla H(\phi(x))| d x \\
& P(\phi)=\int_{\Omega} \frac{1}{2}(|\nabla \phi(x)|-1)^{2} d x
\end{aligned}
$$

The energy $E_{G}$ is defined by:

$$
\begin{gathered}
E_{G}\left(\phi, c_{1}, c_{2}\right)=\int_{\text {inside }(C)}\left|I(x)-c_{1}\right|^{2} H(\phi(x)) d x \\
+\int_{\text {outside }(C)}\left|I(x)-c_{2}\right|^{2}(1-H(\phi(x))) d x
\end{gathered}
$$

Where $c_{1}$ and $c_{2}$ represent mean value of pixels in the region of inside and outside of the contour respectively, $H(\phi)$ is the Heaviside function.

$c_{1}$ and $c_{2}$ are defined as follows:

$$
\left\{\begin{array}{c}
c_{1}=\frac{\int_{\Omega} I(x) \cdot H(\phi) d x}{\int_{\Omega} H(\phi) d x} \\
c_{2}=\frac{\int_{\Omega} I(x) \cdot(1-H(\phi)) d x}{\int_{\Omega}(1-H(\phi)) d \Omega}
\end{array}\right.
$$

$H(\phi)$ is defined as follows:

$$
H_{\varepsilon}(x)=\frac{1}{2}\left[1+\frac{2}{\pi}\left(\arctan \left(\frac{x}{\varepsilon}\right)\right)\right]
$$

The energy $E_{L}$ is defined by: 


$$
\begin{aligned}
& E_{L}\left(\phi, f_{1}, f_{2}\right)= \\
& \int_{\text {inside }(C)}\left|G_{k} * I(x)-I(x)-f_{1}(x)\right|^{2} H(\phi(x)) d x \\
& +\int_{\text {outside }(C)}\left|G_{k} * I(x)-I(x)-f_{2}(x)\right|^{2}(1-H(\phi(x))) d x
\end{aligned}
$$

Where $G_{k}$ is an averaging convolution operator.

$$
\left\{\begin{array}{c}
f_{1}(x) \text { and } f_{2}(x) \text { are defined as follows: } \\
f_{1}(x)=\frac{\int_{\text {inside }(C)}\left(G_{k} * I(x)-I(x)\right) H(\phi) d x}{\int_{\text {inside }(C)} H(\phi) d x} \\
f_{1}(x)=\frac{\int_{\text {outside }(C)}\left(G_{k} * I(x)-I(x)\right)(1-H(\phi)) d x}{\int_{\text {outside }(C)}(1-H(\phi)) d x}
\end{array}\right.
$$

It is worth noticing that the local term $E_{L}$ is designed by finding the best constant approximation of the difference between the averaging filtered image $G_{k} * I(x)$ and the original image $I(x)$ inside and outside of the contour. By subtracting the original image from the local average image, the contrast of the resulted image will be enhanced and the intensity inhomogeneity will be lightened, which are indeed favorable to segment. In our paper, we shall use the intensity difference between averaging filtered image and original image to construct our local region term.

$J\left(\phi, f_{1}, f_{2}\right)$ is defined by:

$$
\begin{aligned}
& J\left(\phi, f_{1}, f_{2}\right)= \\
& \frac{\frac{1}{n_{1}} \int_{\text {in }(C)}\left(I_{0}(x)-f_{1}\right)^{2} H(\phi) d x+\frac{1}{n_{2}} \int_{\text {out }(C)}\left(I_{0}(x)-f_{2}\right)^{2}(1-H(\phi)) d x}{\left(f_{1}-f_{2}\right)^{2}}
\end{aligned}
$$

Where $I_{0}(x)=G_{k} * I(x)-I(x), f_{1}(x)$ and $f_{2}(x)$ are the same as Eq.(14). $n_{1} 、 n_{2}$ are the sum of pixel in the region inside and outside of the contour respectively.

$n_{1}$ and $n_{2}$ are defined as follows:

$$
\left\{\begin{array}{l}
n_{1}=\frac{1}{\int_{\Omega} K_{\sigma} * H(\phi) d x d y} \\
n_{2}=\frac{1}{\int_{\Omega} K_{\sigma} *[1-H(\phi)] d x d y}
\end{array}\right.
$$

Where $K_{\sigma}$ is the Gaussian kernel function, and its expression is as follows:

$$
K_{\sigma}(x-y)=\frac{1}{(2 \pi)^{n / 2} \sigma^{n}} e^{-|x-y|^{2} / 2 \sigma^{2}}
$$

Using the standard gradient descent algorithm to minimize the energy functional in Eq.(7), we derive the level set evolution equation as follows: 


$$
\begin{aligned}
\frac{\partial \phi}{\partial t}= & -\delta(\phi)\left[(1-\omega)\left(\lambda_{1}\left(I(x)-c_{1}\right)^{2}-\lambda_{2}\left(I(x)-c_{2}\right)^{2}\right)\right. \\
& +\omega\left(\lambda_{3}\left(I_{0}(x)-f_{1}\right)^{2}-\lambda_{4}\left(I_{0}(x)-f_{2}\right)^{2}\right] \\
& -\kappa \cdot \delta(\phi) \cdot \frac{\frac{1}{N_{1}}\left(I_{0}(x)-f_{1}\right)^{2}-\frac{1}{N_{2}}\left(I_{0}(x)-f_{2}\right)^{2}}{\left(f_{1}-f_{2}\right)^{2}} \\
& +v \cdot \delta(\phi) \operatorname{div}\left(\frac{\nabla \phi}{|\nabla \phi|}\right)+\mu\left(\nabla^{2} \phi-\operatorname{div}\left(\frac{\nabla \phi}{|\nabla \phi|}\right)\right)
\end{aligned}
$$

Where $c_{1}$ and $c_{2}$ represent mean value of pixels in the region of inside and outside of the contour respectively, and the expressions are the same as Eq.(11). $\delta(\phi)$ is the Dirac function, which can be obtained by the derivative of $H(\phi)$. The expressions of $f_{1}$ and $f_{2}$ are the same as Eq.(14).

The Dirac function is as follows:

$\delta_{\varepsilon}(x)=\frac{1}{\pi} \frac{\varepsilon}{\varepsilon^{2}+x^{2}}$

The implementation of our method is straightforward, and is presented below:

Step 1: Initialize a level set function $\phi$.

Step 2: Update $c_{1}$ and $c_{2}$ using Eq. (11).

Step 3: Update $f_{1}(x)$ and $f_{2}(x)$ using Eq. (14).

Step 4: Update $n_{1}$ and $n_{2}$ using Eq. (16).

Step 5: Update the level set function using Eq. (18).

Step 6: Regularize the level set function with the regularization term.

Step 7: Return to step 2 until the convergence criteria is met.

\section{Experimental Results and Analysis}

This section validates the performance of the proposed model with various synthetic and real medical images from different modalities. We compared our method with ChanVese (CV) method, Local Binary Fitting (LBF) method, Local and Global Intensity Fitting (LGIF) method. In this paper, the initial contours and the final contours are plotted as green contours and red contours, respectively.

All experiments are performed on a PC with Pentium Dual-Core CPU 2. $0 \mathrm{GHz}$ and 2.0 G RAM, using Matlab2012a. We tested the proposed method with the following parameters: $\Delta t=0.1, \mu=1, \lambda_{1}=\lambda_{2}=\lambda_{3}=\lambda_{4}=1$. In order to keep the consistency between information of image and the criterion, we make $\kappa=\eta$. In order to speed up the contour evolution, the initial contour is set to a binary function whose value is 2 . The detailed values of parameters for the different experimental images can be seen from Table 1 .

Table 1. The Parameters of this Paper

\begin{tabular}{|c|c|c|c|c|}
\hline & $v$ & $\omega$ & $\eta$ & $\kappa$ \\
\hline Figure 1 & $0.001 \times 255^{2}$ & 0.98 & 0.98 & 0.000001 \\
\hline Figure 2 & $0.003 \times 255^{2}$ & 0.98 & 0.98 & 0.00001 \\
\hline Figure 3 & $0.003 \times 255^{2}$ & 0.98 & 0.98 & 0.00001 \\
\hline
\end{tabular}




\begin{tabular}{|c|c|c|c|c|}
\hline Figure 4 & $0.001 \times 255^{2}$ & 0.99 & 0.99 & 0.000001 \\
\hline Figure 5 & $0.0008 \times 255^{2}$ & 0.99 & 0.99 & 0.000001 \\
\hline
\end{tabular}

\subsection{Synthetic Images}

In order to demonstrate the capability of our method in dealing with intensity inhomogeneity, we first compare our method with CV, LBF and LGIF to segment a synthetic image with intensity inhomogeneity. Figure1 shows a typical image with intensity inhomogeneity. In addition, the segmentation results with different initializations of the contours are also illustrated in Figure1.

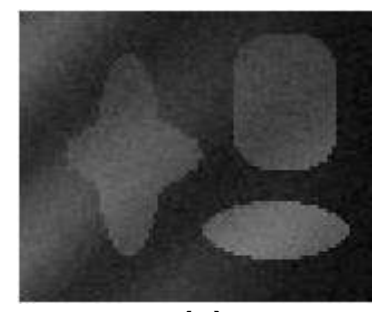

(a)

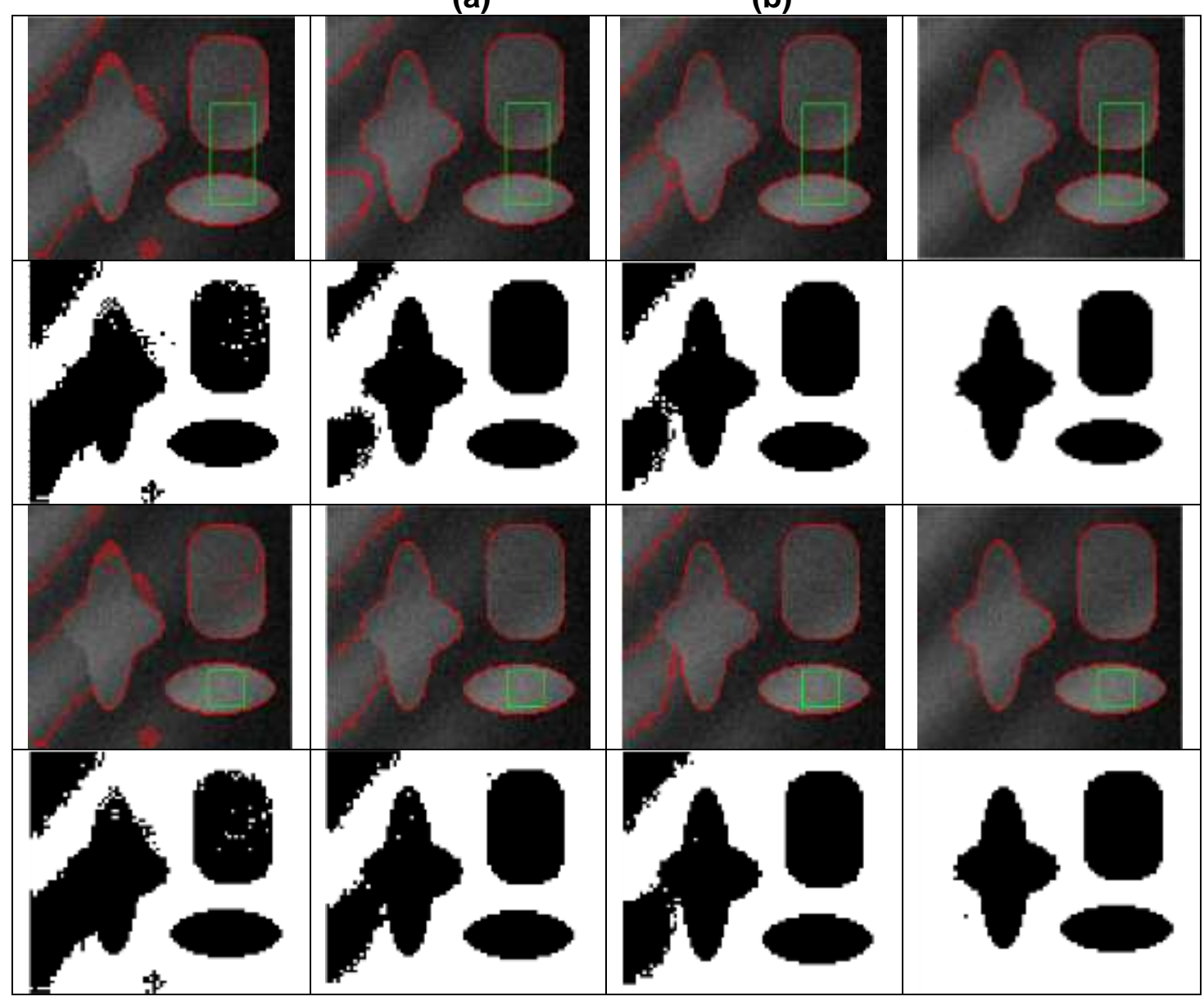

Figure 1. Segmentation Results of CV, LBF, LGIF and Proposed Method for Synthetic Image with Different Initial Contours

In Figure 1, (a) is original image, (b) is the difference image $I_{0}(x)$. The first column: the results of CV model. The second column: the results of LBF model. The third column: the results of LGIF model. The last column: the results of our model. As can be 
seen from Figure1, with different initial contours, CV, LBF, and LGIF models fail to segment the image. By contrast, our method successfully segments the image with different initial contours, as shown in the last column of Figure1.

\subsection{Real Medical images}

Intensity inhomogeneity often occurs in medical images, such as the images shown in Figure2, Figure3, Figure4 and Figure5. All of them are typical images with intensity inhomogeneity. The Figure2 and Figure 3 are two vessel images. In these images, parts of the vessel boundaries are quite weak. The Figure4 and Figure 5 are two brain MR images. As can be seen in these images, some intensities of the white matter (WM) in the upper part are even lower than those of the Gray matter (GM) in the lower part. Generally, in real medical images, the boundaries are quite weak, which renders it a nontrivial task to segment the object from the background.

To illustrate ILGIF model can segment heterogeneous image effectively, the ILGIF model is applied to the real medical image. We compare our method with CV, LBF and LGIF models to segment two real X-ray for vascular images. Figure 2 and Figure3 show the segmentation results. In addition, in the process of experiment, we set different initial contours in the same image, so the segmentation results with different initializations of the contours are also illustrated to prove the robustness of the initial contour in Figure2 and Figure3.

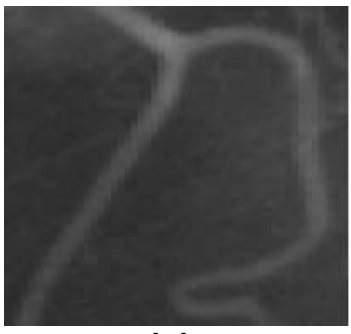

(a)

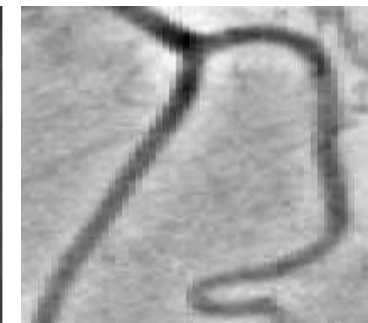

(b)

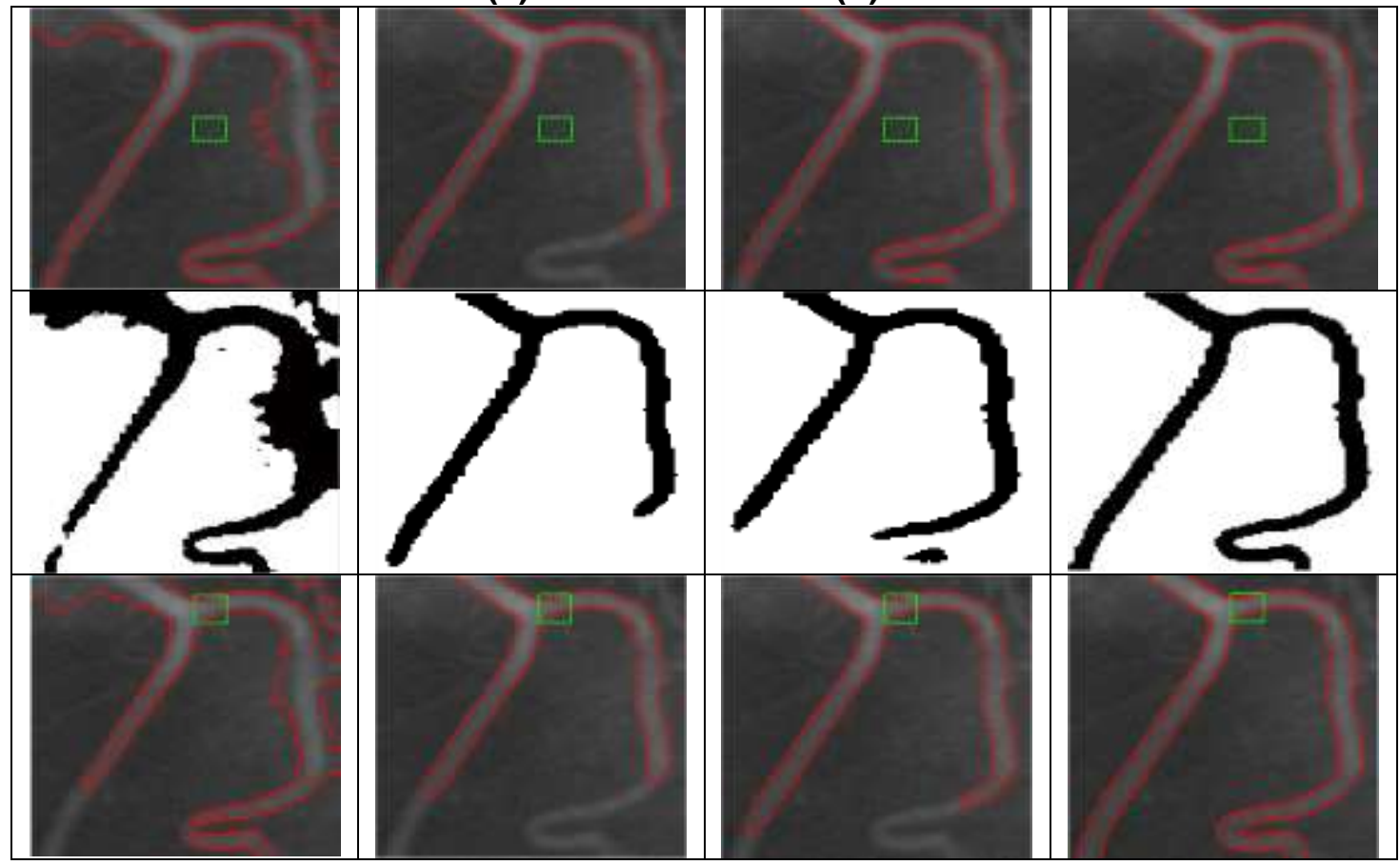




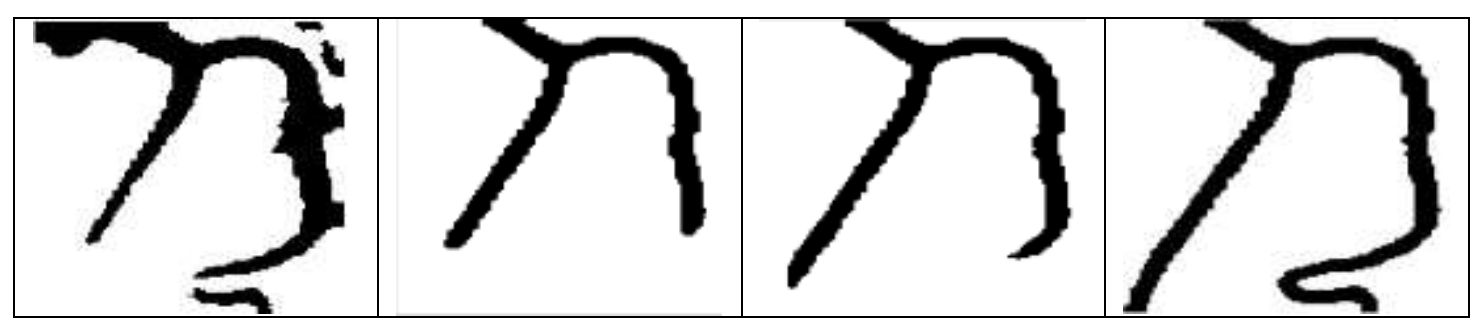

Figure 2. Segmentation Results of CV, LBF, LGIF and Proposed Method for a Blood Vessel Image with Different Initial Contours

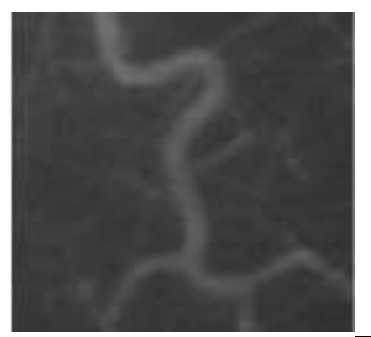

(a)

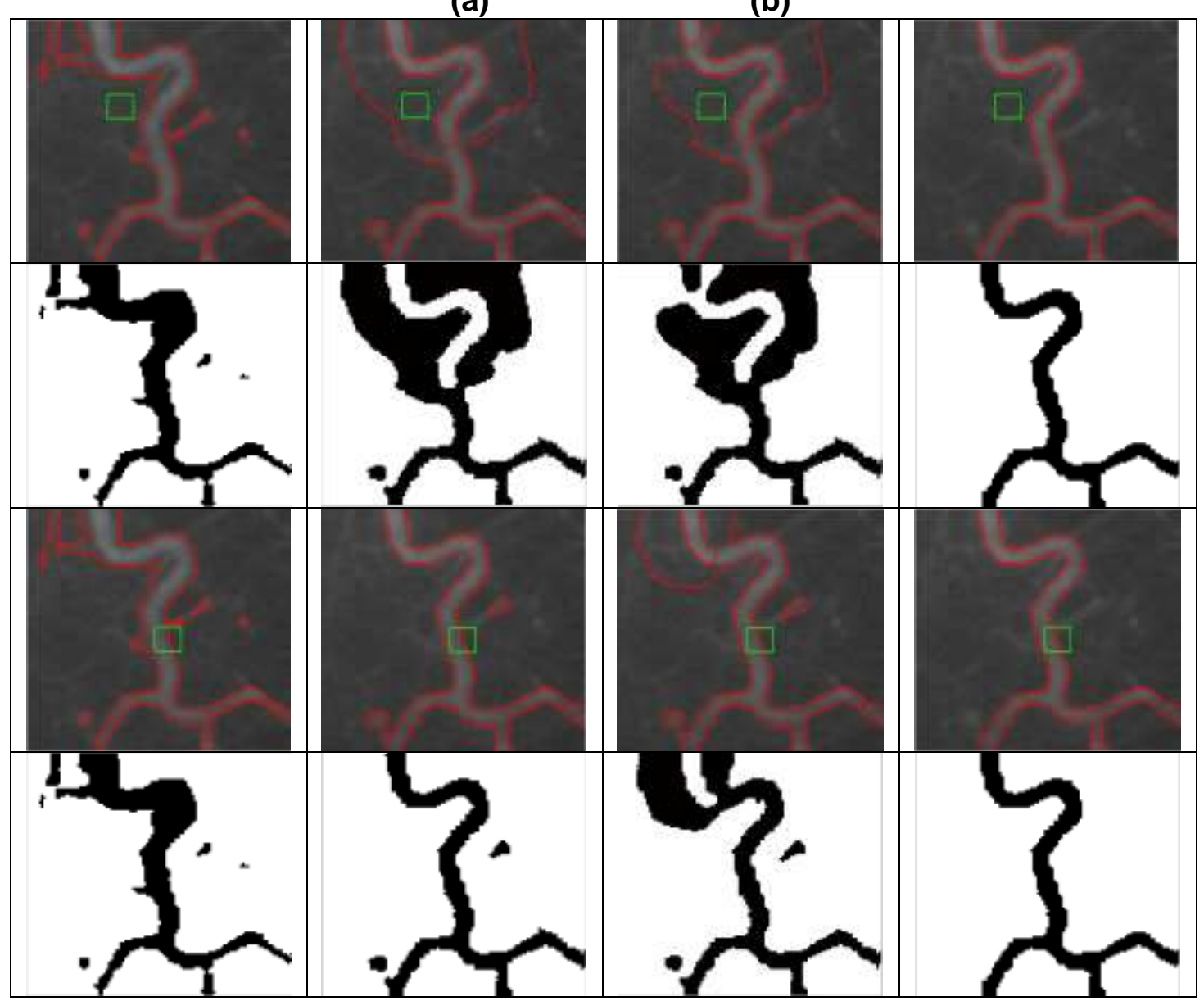

Figure 3. Segmentation Results of CV, LBF, LGIF and Proposed Method for another Blood Vessel Image with Different Initial Contours

In Figure2 and Figure3, (a) is original image, (b) is the difference image $I_{0}(x)$. The first column: the results of CV model. The second column: the results of LBF model. The third column: the results of LGIF model. The last column: the results of our model. 
Figure 2 and Figure 3 show the results of X-ray images of blood vessels. As illustrated in these figures, with different initial contours, CV fails to segment the objects and LBF may and LGIF fail to segment the objects correctly. Specifically, as shown in Figure3, although the initial contours of the first row and the second row are very similar, the segmentation results of LBF are different from each other. It demonstrates that LBF is sensitive to initial contours. Thus, LBF is also sensitive to initial contours. By contrast, the proposed ILGIF model not only gives accurate results but also is robust to different initial contours. In the first row of Figure 2 and the third row of Figure3, for example, even though the initial contours do not contain any foreground objects, our method can still obtain precise segmentation results.

We also use our method to segment images with intensity inhomogeneity. Figure 4 is a brain magnetic resonance image (MRI) with intensity inhomogeneity. In theory, CV cannot segment the details of the white matter. The segmentation results of LBF and LGIF are better than CV, but there are still some segmentation errors. Our method successfully segments the white matter in the same image. It can be observed that our model yields more accurate results than other methods.

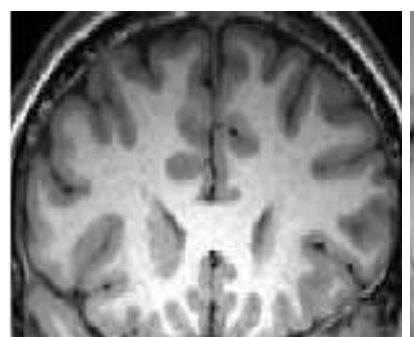

(a)

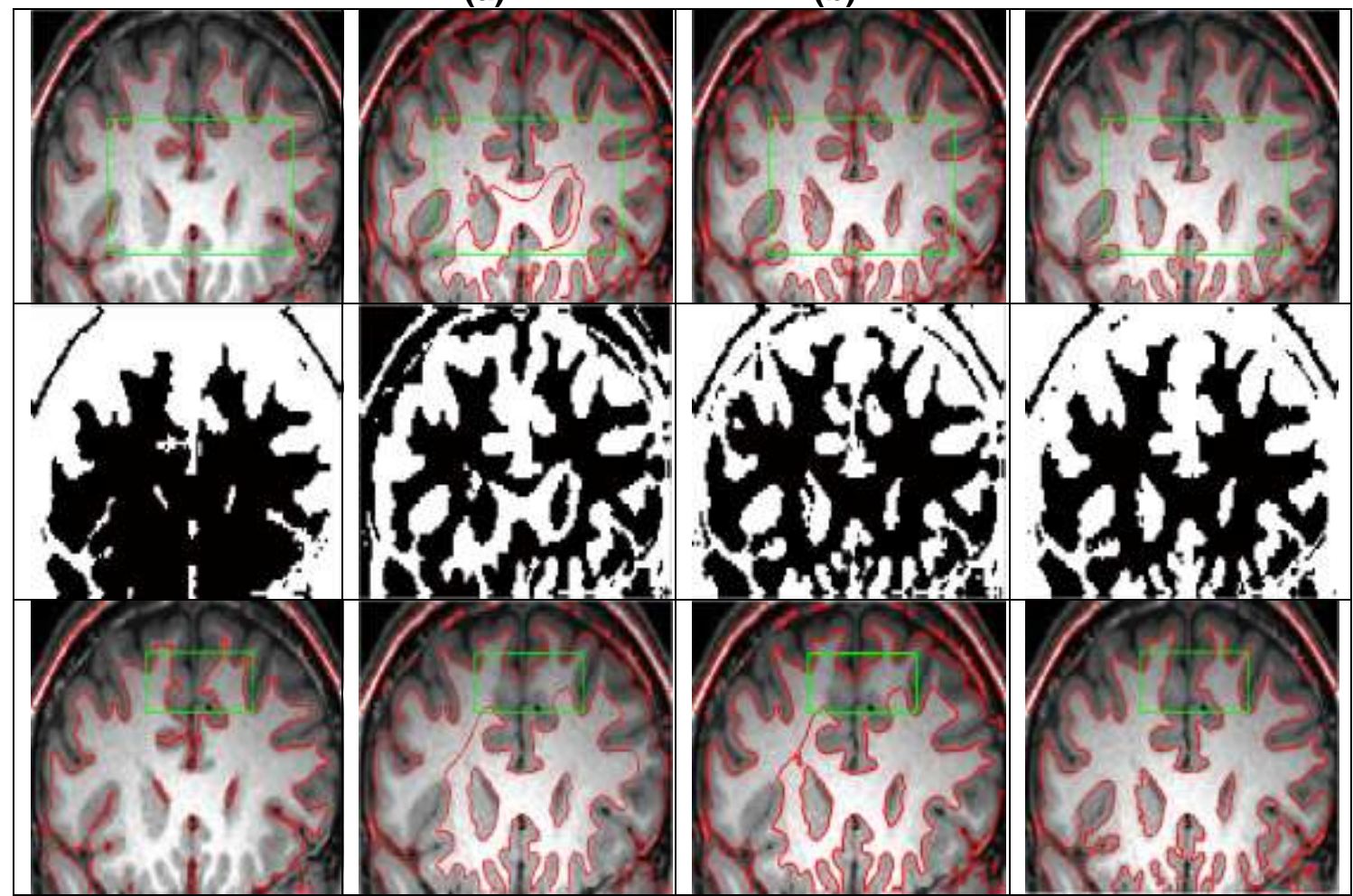

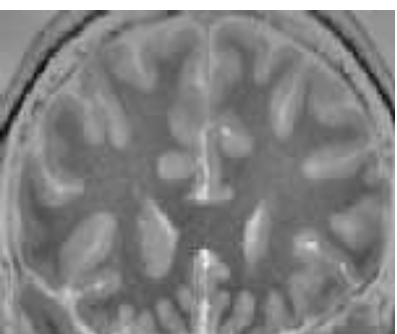

(b) 


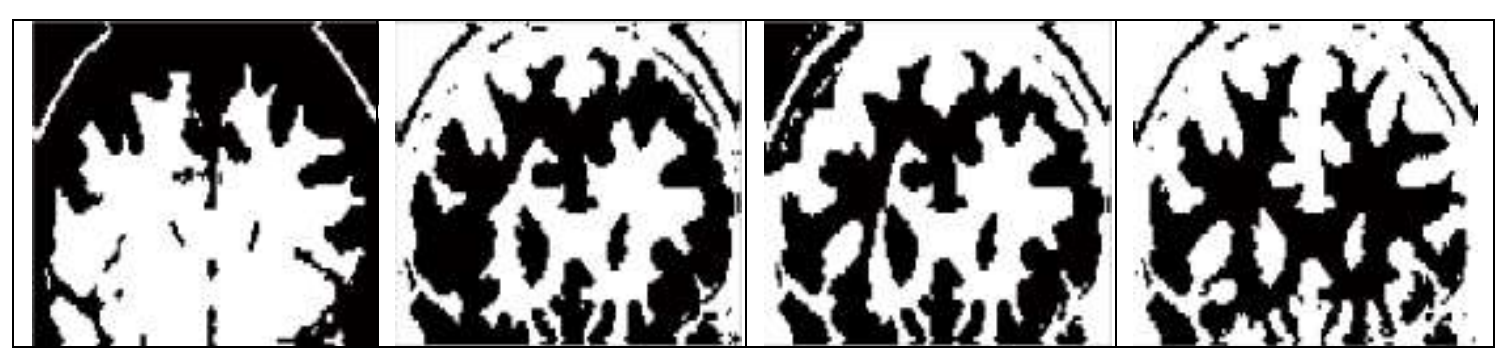

Figure 4. Segmentation Results of CV, LBF, LGIF and Proposed Method for the Brain MR Image

\subsection{Quantitative Experiments}

To illustrate the ILGIF model can improve the accuracy of segmentation results more precisely. We compare our method with $\mathrm{CV}, \mathrm{LBF}$ and LGIF models to segment a brain magnetic resonance imaging (MRI), which size is $174 \times 238$. The experimental results are shown in Figure5 and the quantitative results are shown in Table2. We use the Jaccard similarity (JS) [18] as an index to evaluate quantitatively the segmentation performance of CV, LBF, LGIF models and our method. The JS between two regions $S_{1}$ and $S_{2}$ is calculated as follows:

$J\left(S_{1}, S_{2}\right)=\frac{\operatorname{area}\left(S_{1} \mathrm{I} S_{2}\right)}{\operatorname{area}\left(S_{1} \mathrm{U} S_{2}\right)}$

which is the ratio between the intersectional area of $S_{1}$ and $S_{2}$ and their united area. Obviously, the closer the JS value is to 1 , the more similar $S_{1}$ is to $S_{2}$. In our experiments, $S_{1}$ is the segmented region by the four competing methods, and $S_{2}$ is the ground truth. Due to the randomness of experiments, we run the program 50 times, and then calculated the average of the JS values.
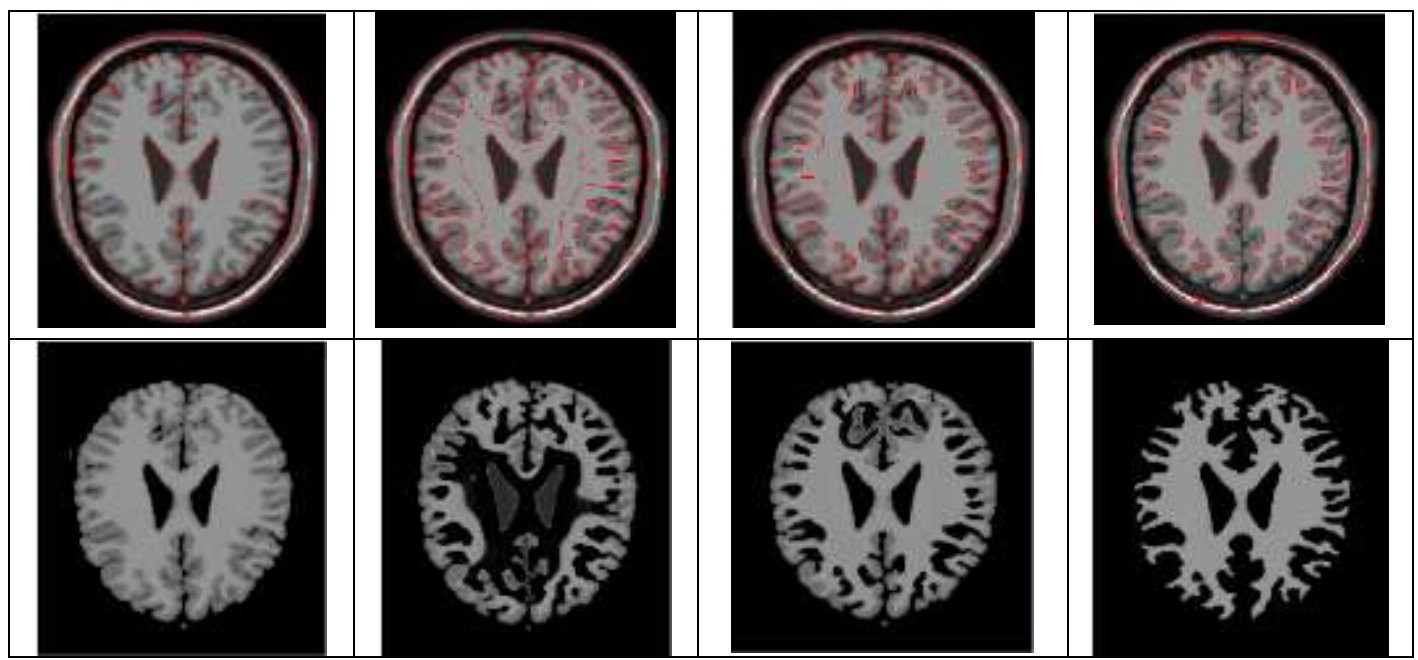

Figure 5. Segmentation Results of CV, LBF, LGIF and Proposed Method for Brain MRI and Extracted Brain White Matter 
Table 2. The JS Values of Brain MRI White Matter Segmentation Results

\begin{tabular}{|c|c|c|c|c|}
\hline & CV & LBF & LGIF & ILGIF \\
\hline JS & 0.309 & 0.603 & 0.704 & 0.896 \\
\hline
\end{tabular}

Figure 5 shows the segmentation results of CV, LBF, LGIF models and our method. Table 2 shows the quantitative comparison results of four models to segment the same brain MRI. We can see that the segmentation result and the segmentation accuracy of the proposed method is superior than the other three methods.

\section{Conclusions}

In this paper, we present a region-based active contour segmentation model with local discriminant criterion for image segmentation. The main contribution of this work lies in that we introduce the local discriminant criterion into level set framework. Comparative experiments on image segmentation show that our method can achieve accurate segmentation results with various initial contours. Introducing discriminant criterion can improve the robustness of our model. However, due to the non-convexity of the energy function, the initialization may still affect the final solution of our model. Although our algorithm shows more robustness to the initialization compared with the traditional algorithms of LBF and LGIF models, it is more reasonable to design a convex energy to drive the evolution of level set function. In addition, for the images with strong bias field, the ILGIF model need further improvements. These weaknesses will be the content of the research in the next step of work about this paper.

\section{Acknowledgments}

The authors would like to thank the Prof. Wang Fengling and anonymous referees for their valuable comments and suggestions, which are very helpful for revising and improving this paper. This research is supported by the Programs, which are Science and Technology Research Key Project (ZD2014129) of Guangxi Province Colleges, and the Fundamental Research Funds for the Hezhou University (2016ZZZK11).

\section{References}

[1] T. B. Borchartt, A. Conci, R. C. F. Lima, R. Resmini, and A. Sanchez, "Breast thermography from an image processing viewpoint: A survey," Signal Processing, vol. 93, (2013), pp. 2785-2803.

[2] D. Cremers, M. Rousson, and R. Deriche, "A review of statistical approaches to level set segmentation: Integrating color, texture, motion and shape," International Journal of Computer Vision, vol. 72, (2007), pp. 195-215.

[3] A. W. M. Kass, D. Terzopoulos, "Snakes: active contour models," International Journal of Computer Vision, (1987), pp. 321-331.

[4] Y. Wu, Y. Wang, and Y. Jia, "Segmentation of the left ventricle in cardiac cine MRI using a shapeconstrained snake model," Computer Vision and Image Understanding, vol. 117, (2013), pp. 990-1003.

[5] V.Caselles, F.Catte, T.Coll, and F.Dibos, "A geometric model for active contour in image processing," Numer. Math., vol. 66, (1993), pp. 1-31.

[6] N. Krishnan and S. N. N. Sujatha, "A fast geometric active contour model with automatic region grid," in Computational Intelligence and Computing Research (ICCIC), 2010 IEEE International Conference on, (2010), pp. 1-8.

[7] V.Caselles, R.Kimmel, and G. Sapiro, "Geodesic active contours," presented at the Processing of IEEE International Conference on Computer Vision'95, Boston, (1995).

[8] X.-F. Wang, D.-S. Huang, and H. Xu, "An efficient local Chan-Vese model for image segmentation," Pattern Recognition, vol. 43, (2010), pp. 603-618.

[9] K. Zhang, L. Zhang, H. Song, and D. Zhang, "Re-initialization free level set evolution via reaction diffusion," IEEE Transactions on Imaging Processing, vol. 22, (2013), pp. 258-271. 
[10] L. A. Vese and T. F. Chan, "A multiphase level set framework for image segmentation using the Mumford and Shah model," International Journal of Computer Vision, vol. 50, (2002), pp. 271-293.

[11] L. Chunming, K. Chiu-Yen, J. C. Gore, and D. Zhaohua, "Implicit Active Contours Driven by Local Binary Fitting Energy," in Computer Vision and Pattern Recognition, 2007. CVPR '07. IEEE Conference on, (2007), pp. 1-7.

[12] C. M. Li, C. Y. Kao, J. C. Gore, and Z. H. Ding, "Minimization of region-scalable fitting energy for image segmentation," Ieee Transactions on Image Processing, vol. 17, (2008), pp. 1940-1949.

[13] T. Brox and D. Cremers, "On the statistical interpretation of the piecewise smooth Mumford-Shah functional," in Scale Space and Variational Methods in Computer Vision, Proceedings. vol. 4485, F. Sgallari, A. Murli, and N. Paragios, Eds., ed, (2007), pp. 203-213.

[14] K. Zhang, H. Song, and L. Zhang, "Active contours driven by local image fitting energy," Pattern Recogn., vol. 43, (2010), pp. 1199-1206.

[15] X. Yue, M. Xie, and L. Li, "Improved LBF Model Combined with Fisher Criterion," in Advances in Future Computer and Control Systems, Vol 1. vol. 159, D. Jin and S. Lin, Eds., ed, (2012), pp. 1-5.

[16] L. Wang, C. Li, Q. Sun, D. Xia, and C.-Y. Kao, "Active contours driven by local and global intensity fitting energy with application to brain MR image segmentation," Computerized Medical Imaging and Graphics, vol. 33, (2009), pp. 520-531.

[17] Y. Yu, C. Zhang, Y. Wei, and X. Li, "Active Contour Method Combining Local Fitting Energy and Global Fitting Energy Dynamically," in Medical Biometrics. vol. 6165, D. Zhang and M. Sonka, Eds., ed: Springer Berlin Heidelberg, (2010), pp. 163-172.

[18] M. Everingham, L. Van Gool, I. Williams, J. Winn and A. Zisserman, "The PASCAL Visual Object Classes (VOC) Challenge”, Int. J. Comput. Vis., vol. 88, no. 2, (2010), pp. 303-338.

\section{Authors}

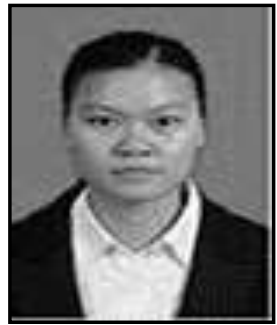

Fangzhen Zhao was born in Guangxi, China, in1987. She received M.E. from Chongqing University, Chongqing, China, in 2014. Her research interests include pattern recognition and image processing. She has been a lecturer in the School of Computer Science, the Hezhou University.

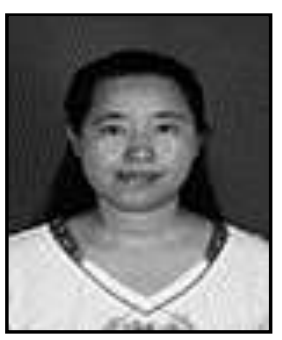

Haiying Liang was born in Jilin, China in 1968. She got her Engineering Ph.D. degree from Northeastern University, Shenyang, China, in 2008. She has been Professor in the School of Computer Science, the Hezhou University. Her research interests include pattern recognition and image processing.

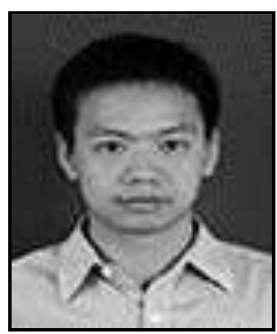

Xianglin Wu was born in Hunan, China in1984. He received M.E. from Guilin University of Technology, Guilin, China, in 2011. His research interests include pattern recognition and image processing. $\mathrm{He}$ has been a lecturer in the School of Computer Science, the Hezhou University.

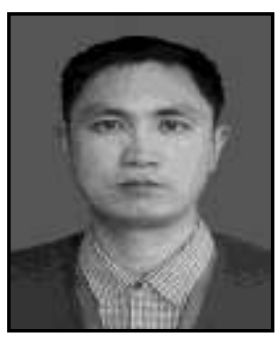

Dehong Ding was born in Hunan, China, in 1975. He received Engineering Ph.D. degree from Hunan Agricultural University, Changsha, China, in 2015. His research interests include pattern recognition and image processing. He has been an Assistant Professor in the School of Computer Science, the Hunan University of Arts and Science. 